\title{
A produção de referências internas (outlinks) e externas (backlinks): um estudo webométrico com a Rede Humaniza SUS
}

\author{
Dalton Lopes Martins \\ Doutor; Universidade de Brasília, Brasília, DF, Brasil; \\ daltonmartins@unb.br \\ Eduardo Alves Silva \\ Mestrando; NOVA IMS, Universidade Nova de Lisboa, Lisboa, Portugal; \\ M20170021@novaims.unl.pt
}

\begin{abstract}
Resumo: Compreender a apropriação social em um ambiente web traz benefícios para o entendimento da dinâmica social e das práticas sociais dos coletivos humanos interconectados. A presente pesquisa se refere a Rede Humaniza SUS (RHS), analisada em um estudo a respeito dos links de saída (outlinks) que são apresentados como endereços externos nas postagens da rede, e os links de retorno (backlinks) que geram acessos provenientes de sites externos. A partir do uso da webometria foi feito o uso da metodologia de Análise de Rede Sociais (ARS) que implementa um aspecto visual aos dados de forma a facilitar sua compreensão, e demonstrando como a rede é percebida como uma fonte de informação por diferentes instituições, muitas delas na área da saúde, assim como essas instituições são acessadas e referenciadas pelos usuários da rede. Os resultados apontam intenso uso de mídias sociais, sites institucionais e webmail como sendo as referências que socializam conteúdos produzidos pela rede e os sites institucionais e governamentais como sendo as fontes de citação dos usuários da rede.
\end{abstract}

Palavras-chave: Rede Humaniza SUS. Webometria. Análise de Redes Sociais.

\section{Introdução}

O presente trabalho é uma extensão do trabalho apresentado no $6^{\circ}$ Encontro Brasileiro de Bibliometria e Cientometria ( $\left.6^{\circ} \mathrm{EBBC}\right)$, com o título Apropriação Social de Redes de Relacionamento: a webometria como ferramenta de percepção da produção de referências internas (outlinks) e externas (backlinks $)^{1}$, tendo como diferencial uma melhor apresentação dos resultados obtidos pela pesquisa e um embasamento teórico alargado a respeito do tema a que se remete, gerando uma melhor compreensão do propósito deste estudo. 
A bibliometria é um amplo campo de pesquisa que pode se conectar com a cientometria em diferentes pontos, assim como em outras áreas de estudo, como é o caso da webometria, que se trata de um campo de estudo o qual se propõe a compreender a web como fenômeno informacional, levando em consideração sua visibilidade, impacto e interação. Esse campo faz uso das informações de âmbito virtual que acabam por representar as relações de sites na web por meio de diferentes atos e significados de produção de links entre eles.

A webometria é definida como "o estudo dos aspectos quantitativos da construção e uso de recursos de informação, estruturas e tecnologias na web utilizando-se de abordagem bibliométrica e informétrica". (BJORNBORN, 2004, p. 12, tradução nossa).

Entende-se que, assim como para os estudos bibliométricos, a webometria pode oferecer percepções de estratégias específicas de como determinados sites na web se relacionam entre si, trazendo à tona informações a respeito de como referenciam outros sites externos ao seu ambiente, os outlinks, acionando fontes de informação que se tornam reconhecidas e validadas por seus usuários (GOUVEIA, 2012; SILVA, 2016).

Também podem trazer informações relevantes como determinados sites se referenciam internamente, ressaltando informações que se tornam apropriadas e explicitamente resignificadas por novas citações da própria produção interna de um site, os inlinks (VANTI, 2005).

Além do mais, estudos de natureza webométrica podem oferecer recursos analíticos que ajudam a perceber como os sites são percebidos externamente a si próprios, por meio da análise de backlinks, na qual o mundo externo a um site o reconhece explicitamente por meio de links de suas páginas que direcionam os usuários para esse site específico. Um universo de estratégias de análise de referenciamento se torna disponível ao pesquisador para que o mesmo possa entender os processos de apropriação social de um site por meio de como seus links são produzidos.

O presente trabalho tem por objetivo apresentar um estudo webométrico em andamento sobre uma importante rede social pública brasileira, criada e mantida no âmbito da Política Nacional de Humanização da Saúde, a Rede Humaniza SUS (RHS) ${ }^{2}$. Trata-se da primeira rede social criada a partir de uma 
política pública brasileira e a mais antiga ainda em atividade, a rede está no ar desde o ano de 2008, contando com mais de 30.000 usuários espalhados pelo Brasil em torno da discussão e produção de conteúdo a respeito da humanização da saúde. A pergunta de pesquisa que norteia o presente trabalho é quais as referências externas dos usuários, seus outlinks, e como essas referências percebem a rede por meio de links de retorno, os backlinks. Tem-se como hipótese que as referências externas são elementos de autoridade reconhecidas pelos usuários e que as referências de retorno são mais fortemente pautadas por ambientes web de socialização.

Destaca-se que o uso de redes e mídias sociais criados por políticas públicas ainda é um fenômeno em intensa análise e pesquisa pelo mundo afora. Há muitas teorias que avaliam as possibilidades de exercício de novas formas de cidadania e democracia intermediadas pelas novas tecnologias digitais (ALCÂNTARA; TUZZO, 2014; SANTOS, 2013), enquanto outras pesquisas apontam estudos de caso relevantes para que se avance de forma consistente o conhecimento sobre o que pode e como podem se criar novas experimentações nesse sentido (MORIGI; MASSONI; SENA, 2016; SILVA, 2013). No entanto, há ainda pouco consenso e teorias bem formuladas que ajudem a explicar os sentidos de uso e potenciais conceituais de como essas redes são de fato apropriadas pelas pessoas e como são contextualizadas do ponto de vista informacional em seu cotidiano de uso.

Dessa maneira, dado o conjunto significativo de dados que estão à disposição que tratam da RHS, a sua importância política e, sobretudo, a sua série histórica relatando dados de relacionamento entre usuários de já mais de uma década, a motivação para a realização da presente pesquisa é poder apontar, por meio de técnicas webométricas, formas de se perceber como os usuários dessa rede referenciam sites externos a rede e como ela própria é referenciada de fora de seu ambiente. Esta pesquisa visa identificar tendências de referenciamento que podem apontar dinâmicas de relacionamento social que ajudem a explicar como esses ambientes podem ser incorporados e experimentados na dimensão das políticas públicas. 


\subsection{Webometria, Weblinks e a Rede Humaniza SUS}

A webometria apresenta um método de estudo que cruza o quantitativo com o qualitativo, sendo capaz de medir informações disponibilizadas na web através do uso de conexões por weblinks. Ao compreender as informações, é possível visualizar conexões que podem ser consideradas sociais entre sites na rede.

Almind e Ingwersen podem ter sido os primeiros autores a utilizarem o termo "webometrics" em 1997. Na língua portuguesa, nomeadamente no Brasil o termo utilizado é webometria pelo fato de estar relacionado à bibliometria e infometria.

Segundo Thewall et al. (2003, p. 2), conforme citado por Vanti (2007, p. 64):

[...] a Webometria trata dos aspectos quantitativos tanto da construção quanto do uso da Web, compreendendo quatro áreas principais de pesquisa: análise de conteúdo das páginas web; análise da estrutura dos weblinks; análise do uso da web [...] e análise de tecnologias na Web.

A webometria é uma área de estudo que se aproxima de outros campos, como a bibliometria e outras métricas, como apresentando na Figura 1, na qual se vê a relação entre os diversos outros campos e a webometria.

Figura 1 - Relação entre os campos/subcampos das métricas

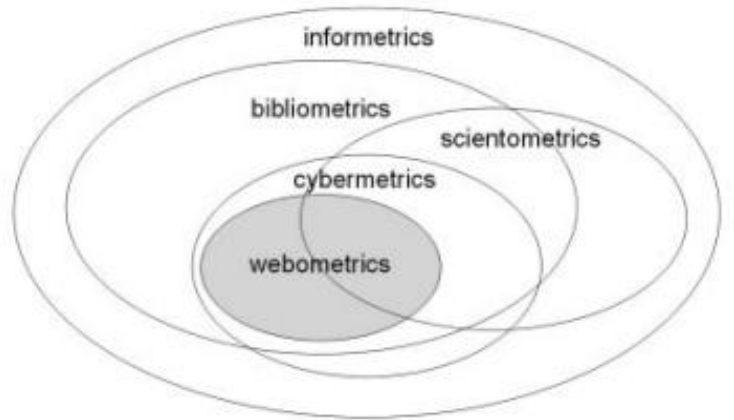

Fonte: Björneborn e Ingwersen (2004).

O estudo sobre a webometria tem sido largamente abordado pelo mundo afora, trazendo conceitos já utilizados por outros campos, mas que agora se aplicam a $W e b$, o que gera novos valores aos estudos qualitativos a respeito de 
relações entre ambientes que se encontram na rede, sendo que a "webometria e a cybermetria são os termos mais utilizados atualmente em bibliometria e ciências da informação para esse campo de pesquisa emergente". (BJÖRNEBORN; INGWERSEN, 2004, p. 2).

A relação entre o estudo de weblinks e a webometria é apresentada como um fator de importância, uma vez que a partir do número weblinks em uma página, podem ser desenvolvidos estudos a respeito da densidade desses weblinks (ALMIND, 1997; IGWERSEN, 1997), sendo uma forma de mensurar a relação entre o tamanho/importância de uma página/site na web e a quantidade de weblinks que ela aponta.

Os weblinks são apresentados como um conceito básico no espaço hipertextual, que permeia toda a web e a atual construção de páginas nesse ambiente, dado que é a partir dos weblinks que as correlações entre diferentes tipos de conteúdo e informações reproduzem diferentes produtos. Esses produtos podem ser diversos, como uma imagem, um vídeo ou uma outra página web; esses diferentes produtos aos quais os weblinks se conectam podem ser considerados como conexões dentro da rede. Essas conexões acabam por demonstrar as relações entre as diversas páginas e como essas se complementam gerando uma rede entre si, exercendo impacto de acordo com o peso de cada conexão.

Segundo a visão de Paul Otlet, citado por (WRIGHT, 2003), um documento tem três dimensões, sendo sua terceira o contexto social. Os weblinks, por sua vez, podem ser considerados como uma relação social na web, que se estabelece a partir de documentos criados nesse contexto, seja um texto em um site ou vídeo, o contexto social tem reflexo nas relações que são produzidas a partir da interação de usuários com um texto criado em um blog, ou na relação desse texto com outros documentos ou páginas web.

O desenvolvimento da webometria levou a construção de uma terminologia para os diferentes tipos de weblinks. Alguns desses termos como outlinks e inlinks são usados comumente em ciências da computação (BJÖRNEBORN, 2004). Na Figura 2, tem-se uma perspectiva visual dos diferentes tipos de relações entre weblinks e páginas ou sites; a partir da 
Dalton Lopes Martins e Eduardo Alves Silva

imagem, as letras podem ser consideradas páginas ou sites e as setas representam as formas de conexão, e sua descrição na Tabela 1.

Figura 2 - Relação básica entre links, Björneborn (2004)

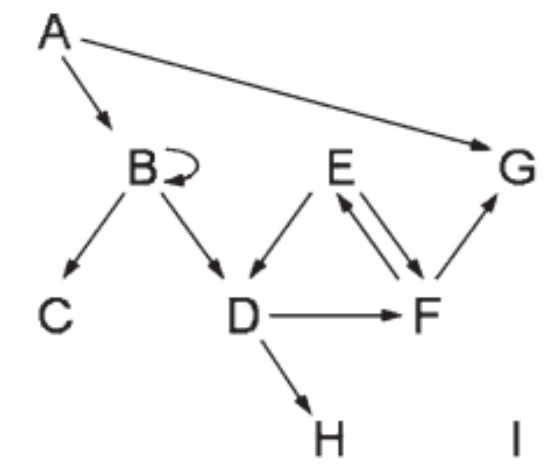

Fonte: Adaptado de Björneborn (2004).

Tabela 1 - Terminologia básica de links Björneborn (2004) para a Figura 2

- B recebe um inlink de A: se relaciona com a citação em bibliometria

- B tem um outlink para C: referência

- B tem um selflink: autocitação

- C e D tem coinlinks de B: cocitação

- B e E tem cooutlinks de D

Fonte: Adaptado de Björneborn (2004).

A partir desse tipo de informações, é viável a construção de diferentes caminhos para a compreensão da interação na web, como é o caso de estudo com a RHS, com informações referentes a como a rede se cita (inlinks), como outros sites são citados na rede (outlinks) e quais os caminhos traçados pelos usuários que visitam a rede (backlinks).

Vale resaltar que a RHS é um sistema de blog coletivo criado em 2008, no qual os usuários se cadastram e passam a ter o direito de publicar postagens que passam então a serem moderadas de forma coletiva por outros usuários do ambiente. Dessa maneira, as postagens são produzidas de forma descentralizada, não havendo uma única instituição responsável pela produção de seu conteúdo. A dinâmica, portanto, permite que os usuários possam fazer links das mais variadas origens e conteúdos, fornecendo conteúdos valiosos para que se possa compreender de maneira significativa qual é o repertório de referências que 
esses usuários possuem quando usam esse recurso de citarem sites externos como forma de embasar seu conteúdo publicado.

Da mesma maneira, a rede é também referenciada externamente por diversos outros sites pela internet, tornando-se essa informação uma dimensão relevante de compreensão de como a rede é lida e citada por outras fontes de informação em rede. É com base na compreensão dessa dinâmica que a presente pesquisa se organiza.

\section{Metodologia}

Para a obtenção dos resultados esperados para análise e compreensão do impacto das postagens da RHS, foi realizada a extração de dados dos outlinks a partir da base de dados da RHS por meio da análise de conteúdo das postagens, enquanto a partir da ferramenta Google Analytics foram extraídos os backlinks, por meio da análise da série histórica armazenada por essa ferramenta desde 2008.

No que diz respeito à coleta e organização dos dados relacionados aos outlinks, utilizou-se um script na linguagem de programação Python, onde o mesmo acessa as postagens da RHS a partir do seu banco de dados, captando assim todos os links existentes nessas postagens a partir do código apresentado no Quadro 1.

Quadro 1 - Script em Python para a extração de outlinks das páginas RHS

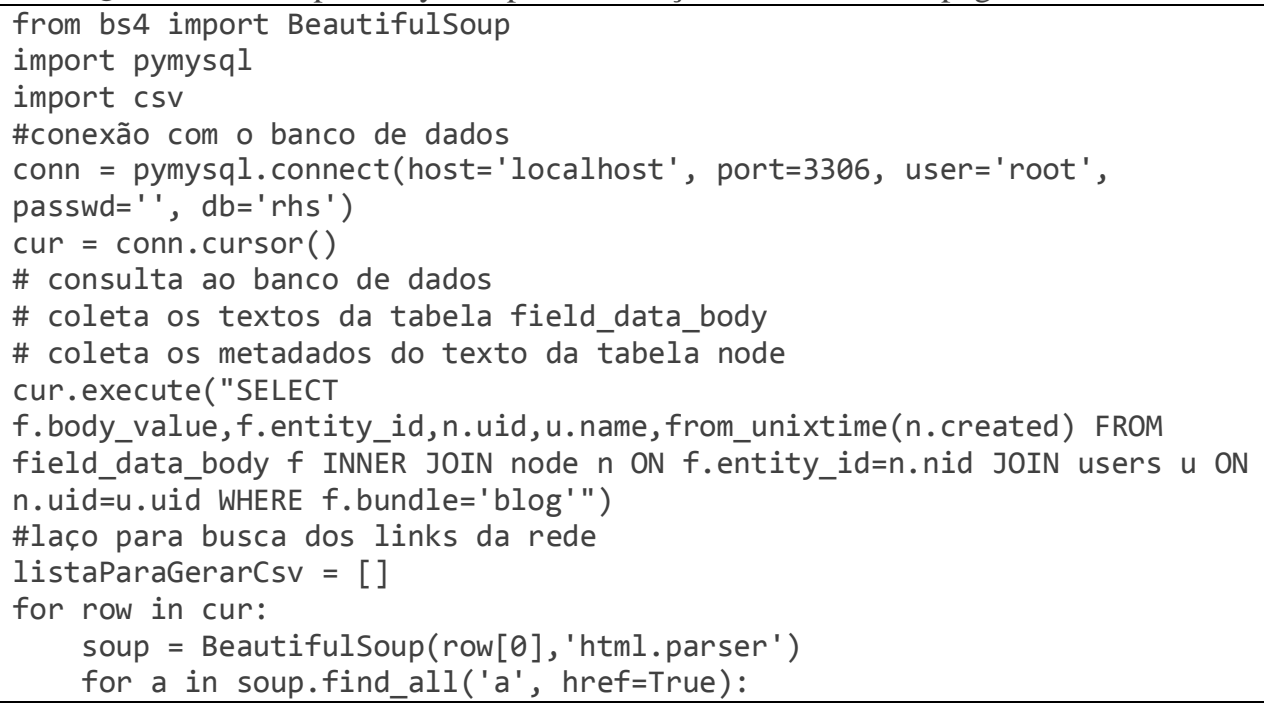




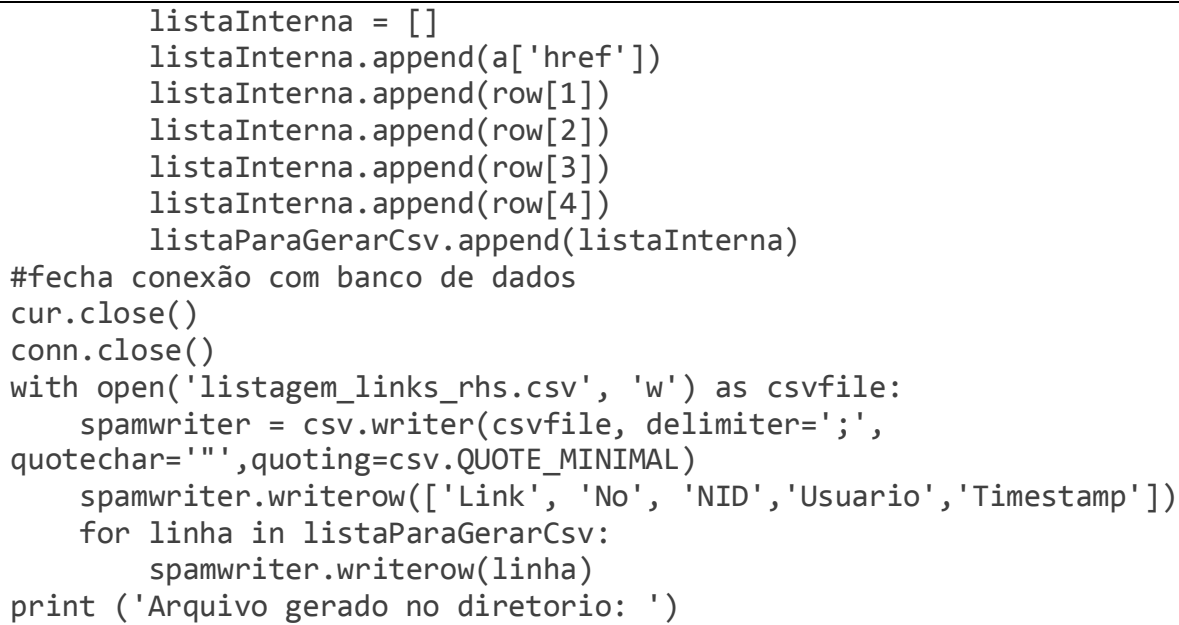

Após a obtenção dos dados foi necessário efetuar sua padronização para uso. Ao cruzar esses dados com os dados do banco de dados, é possível definir o número total de links por postagem, e por sua vez o número de links gerado pelos usuários que contribuíram com tais postagens.

Os backlinks por sua vez tiveram o tratamento de dados diferenciado, uma vez que se trata da reprodução dos caminhos traçados pelos visitantes da RHS para acessá-la, com base nisso, se utilizou o Google Analytics da RHS juntamente com o Google Query Explorer. Essa ferramenta possibilita a extração seletiva de dados do Google Analytics, mas para tal foi necessária uma melhor compreensão da disposição desses dados dentro do Google Analytics. Para esta análise, os dados recuperados foram o link do site ou página pelo o qual o visitante chegou a RHS, a página da RHS acessada a partir desse link, a quantidade de usuários provenientes desse link e o tempo estimado que o usuário se manteve na RHS. Os dados foram extraídos em outubro de 2017. 


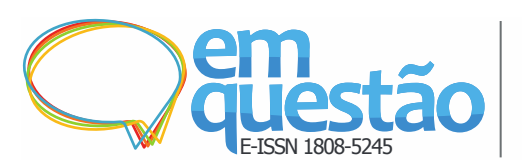

\section{A produção de referências internas (outlinks) e externas (backlinks): um estudo webométrico com a Rede \\ Humaniza SUS \\ Dalton Lopes Martins e Eduardo Alves Silva}

Figura 3 - Google Query Explorer usado para a extração de dados do Google Analytics

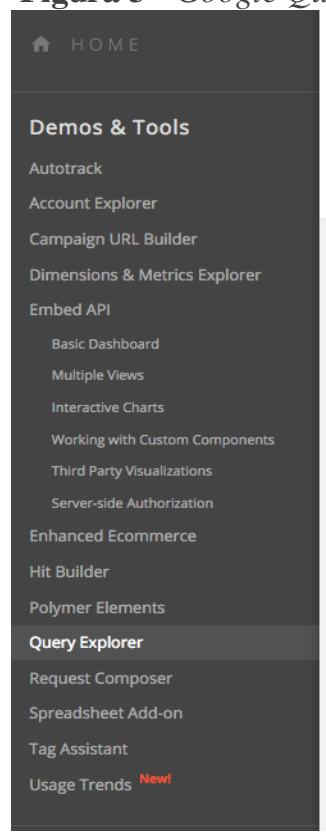

(i) Google Analytics | Demos \& Tools

\section{Query Explorer}

\section{Overview}

Sometimes you just need to explore. This tool lets you play with the Core Reporting APl by building queries to get data from your Google Analytics views (profiles). You can use these queries in any of the client libraries to build your own tools.

Select a view

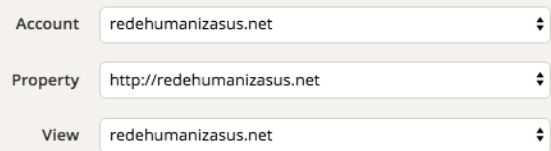

Set the query parameters

* ids ga:8696380

* start-date $\quad 2008-01-01$ $\square$

Fonte: Google Query Explorer (2017).

A extração dos dados e criação de correlações com as postagens da RHS se deu em sua maioria a partir do uso do número de identificação de cada uma das postagens, que é apresentado no banco de dados.

Após a obtenção da totalidade de links e compreensão dos seus padrões foi possível criar uma categorização entre backlinks e outlinks, essa etapa foi produzida levando em consideração as redes sociais mais comuns entre os links, e seus domínios. Por exemplo, domínios que contenham wordpress ou blogspot em seu endereço são considerados $b \log s$, domínios que tenham algum vínculo institucional com universidades levam a categoria "instituição" atribuída. No Quadro 2 são apresentadas as respectivas categorias e o que representam.

Quadro 2 - Categorização de outlinks e backlinks da RHS

\begin{tabular}{|c|c|}
\hline Categorias & Representação \\
\hline$B \log$ & Links do Blogspot ou Wordpress.com \\
\hline Governamental & Todos os links com o domínio.GOV \\
\hline Instituições & Links de Instituições de Ensino e Universidades \\
\hline Periódico & Links de periódicos científicos \\
\hline Portal de Notícias & Portais de notícias on-line \\
\hline Rede Social & $\begin{array}{c}\text { Todos os links relacionados a redes sociais como, Twitter, Facebook, } \\
\text { Youtube. }\end{array}$ \\
\hline Wikipédia & Links da Wikipédia \\
\hline Webmail & Links que representam e-mails comuns ou institucionais \\
\hline Site & $\begin{array}{c}\text { Todos os links que não se enquadram em nenhuma das categorias } \\
\text { anteriores }\end{array}$ \\
\hline
\end{tabular}

Fonte: Dados da pesquisa (2017). 
Após a categorização, e com todos os dados limpos e padronizados, foi possível gerar as correlações entre as postagens e os weblinks, o que permitiu que as páginas e conexões representados no estudo webométrico fossem visualizados como uma rede de conexões. Para tal, foi feito o uso da análise de redes sociais e quatro indicadores de rede, apresentados no Quadro 3, que conseguem demonstrar o impacto dos backlinks e outlinks na RHS.

Quadro 3 - Indicadores de Análise de redes sociais.

\begin{tabular}{|c|c|}
\hline Indicadores & Significado \\
\hline Nós & Representação de atores que fazem parte da rede \\
\hline Arestas & Número de relações existentes entre os atores \\
\hline Centralidade de Grau & Ator da rede com maior atividade ou relações, em relação aos demais \\
\hline Clusters & Conjunto de nós que fazem parte de um mesmo grupo \\
\hline \multicolumn{2}{|c}{ Fonte: Wasserman e Faust (1994); Cherven (2015). }
\end{tabular}

\section{Resultados}

Com o tratamento dos dados que remontam ao período entre fevereiro de 2008 e outubro de 2017, obteve-se um total de 4.609 outlinks da rede e 1.203.191 backlinks. Percebe-se aqui a grande quantidade de dados que se tornam disponíveis para análise e estudo.

A Figura 4 mostra a comparação entre a quantidade e o tipo de links da RHS, normalizados em porcentagem, nota-se que $40 \%$ dos links da RHS se encontra na categoria "Site", seguida da categoria "Rede social"

Figura 4 - Porcentagem de links por categoria de outlinks e backlinks da RHS

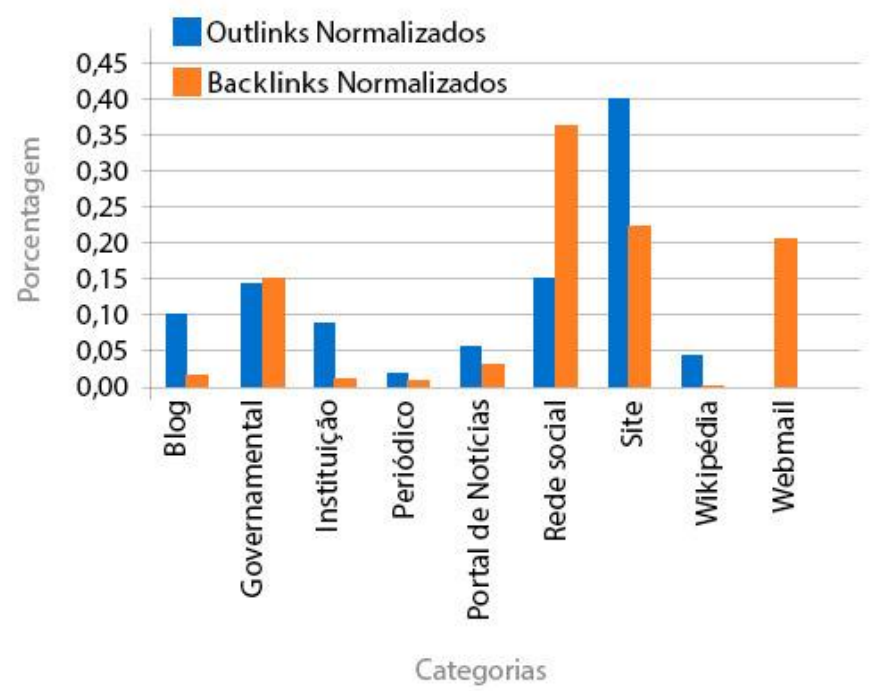

Fonte: Dados da pesquisa (2017). 
Percebe-se que as categorias "redes sociais" e "webmail" possuem um relevante papel no que diz respeito a referenciar a rede, uma vez que essas referências dizem respeito aos backlinks, o que em suma mostra a difusão de informação e compartilhamento de links da rede na web e o retorno de usuários por esse caminho. São fontes de socialização da rede. Chama a atenção o papel desempenhado pelo webmail, dado que não se esperava sua alta importância. Infere-se, a partir disso, a relevância da socialização por meio desses mecanismos e a formação de redes privadas de usuários que se articulam em torno dos conteúdos produzidos pela RHS.

Por outro lado, observa-se que em relação aos outlinks a categoria com maior presença é "Site", isso mostra que majoritariamente as postagens da RHS referenciam sites institucionais. Essa dinâmica já era esperada, dado que a discussão em torno da saúde pública se faz ao redor de editais, normativas e informações oficiais, entre outros elementos que constituem as fontes oficiais institucionais de informação.

Uma vez que os links estejam categorizados e normalizados, é possível identificar no Quadro 4 quais backlinks e outlinks produziram mais links na rede.

Quadro 4 - Dez domínios de maior frequência de outlinks e backlinks na RHS

\begin{tabular}{|c|c|c|c|}
\hline Backlinks & Quantidade & Outlinks & Quantidade \\
\hline Facebook.com & 677.697 & Facebook.com & 275 \\
\hline Portal.saude.gov.br & 110.796 & Pt.wikipedia.org & 194 \\
\hline Google.com.br & 26.159 & www.youtube.com & 191 \\
\hline Mail.terra.com.br & 13.212 & Ulbra-to.br & 187 \\
\hline Pensesus.fiocruz.br & 9.785 & Saudepublica.sul21.com.br & 146 \\
\hline Mail.uol.com.br & 9.726 & Bvsms.saude.gov.br & 94 \\
\hline Redehumanizasus.net & 8.468 & www.icit.fiocruz.br & 78 \\
\hline Potalses.saude.sc.gov.br & 6.450 & G1.globo.com & 75 \\
\hline Bvms.saude.gov.br & 4.793 & Docs.goog.com & 67 \\
\hline Blog.saude.gov.br & 3.642 & www.scielo.br & 65 \\
\hline
\end{tabular}

Fonte: Dados da pesquisa (2017).

Percebe-se que o Facebook se destaca, sendo o domínio mais referenciado, tanto como elemento de socialização no qual circulam links redirecionando os usuários para dentro da RHS, quanto como referência que são citadas por usuários da RHS na produção de suas postagens. Isso, vale reforçar, em termos de resultado individual se mostra um dado surpreendente. Não se esperava o Facebook como fonte de citação de informações para a rede, já que 
as publicações da RHS em sua maioria estão relacionadas a sites ou notícias sobre o tema saúde, ou seja, textos muito técnicos e focados em apenas uma temática. Mas nota-se que os usuários compreendem o que ali circula como também um conteúdo que merece referência e assim ele passa a ser incorporado nos textos coletivamente construídos pelos usuários da rede. A socialização das fontes é característica marcante desse resultado.

Já quando se observa os resultados dos backlinks, se percebe três aspectos importantes. O primeiro é o papel do mecanismo de busca do Google, que indexa os conteúdos produzidos pela rede tornando-se um site de redirecionamento de usuários. O segundo são os sites da área da saúde, como o site oficial do Ministério da Saúde $^{3}$, da Fiocruz ${ }^{4}$ e da BVMS $^{5}$, que são de reconhecidas instituições da área e que referenciam a RHS em seus próprios conteúdos, legitimando a rede como fonte de informação relevante para sua área temática. O terceiro são os servidores de e-mails, como o Terra e o UOL, que podem ser compreendidos como ferramentas de socialização, servindo como mecanismos de circulação de links da rede.

Na observação dos outlinks, a dinâmica encontrada é relativamente diferente. Aqui destacamos também três movimentos relevantes. O primeiro é o papel das mídias sociais como fontes de informação; o já citado Facebook e o próprio Youtube utilizados para enriquecer conteúdos on-line. O segundo é o papel dos sites institucionais da área, que também passam a ser fontes de informações para seus usuários, tais como a própria Fiocruz e novamente a BVMS. O terceiro são sites de notícia, que se diferenciam dos principais backlinks, por serem utilizados pelos usuários como fontes comentadas em suas postagens.

\subsection{Visualização de Outlinks e Backlinks}

Para visualizar os dados e resultados alcançados foi utilizado a metodologia de Análise de Redes Sociais (ARS), que se enquadra na visualização de dados webométricos, dado que os links podem ser vistos como nós que se conectam entre si. Ao aplicar o conceito de ARS é possível perceber os nós mais significativos entre os backlinks e outlinks, ou mesmo ter uma visão mais ampla dessas conexões. 
A Figura 5 representa a rede de outlinks gerada a partir dos dados coletados na RHS. Essa rede tem 885 agrupamentos, sendo que 23 desses agrupamentos se apresentam bem definidos e são identificados por diferentes cores, sendo que foram produzidos um total de 4.244 nós e 3.867 arestas (relações entre os nós). Muito embora a rede se mostre granulada em suas camadas mais externas, há muitos nós que se apresentam afastados ou com relações fracas entre si.

Figura 5 - Rede de Outlinks da RHS

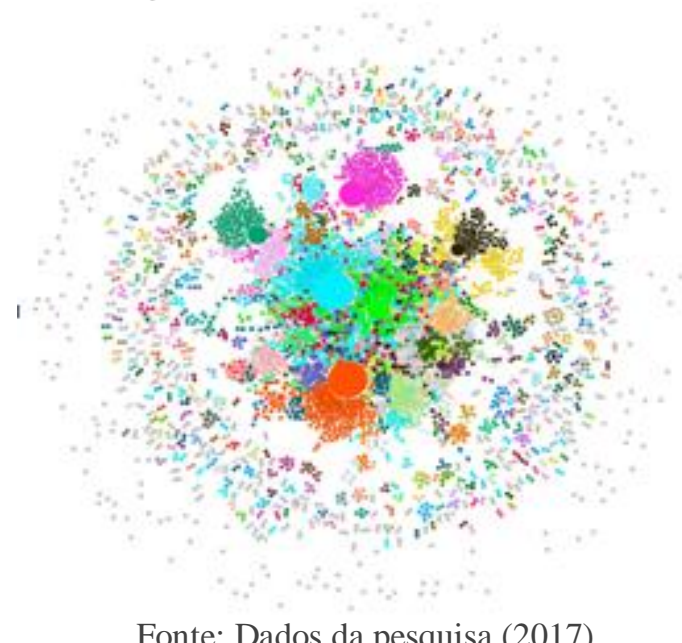

Fonte: Dados da pesquisa (2017).

Na Figura 6 podem ser visualizados os outlinks que aparecem nas postagens da RHS com uma frequência igual ou maior que dez vezes. A imagem que se verifica é, na verdade, um zoom na Figura 5.

Figura 6 - Outlinks que aparecem com uma frequência >= 10 nas Postagens da RHS.

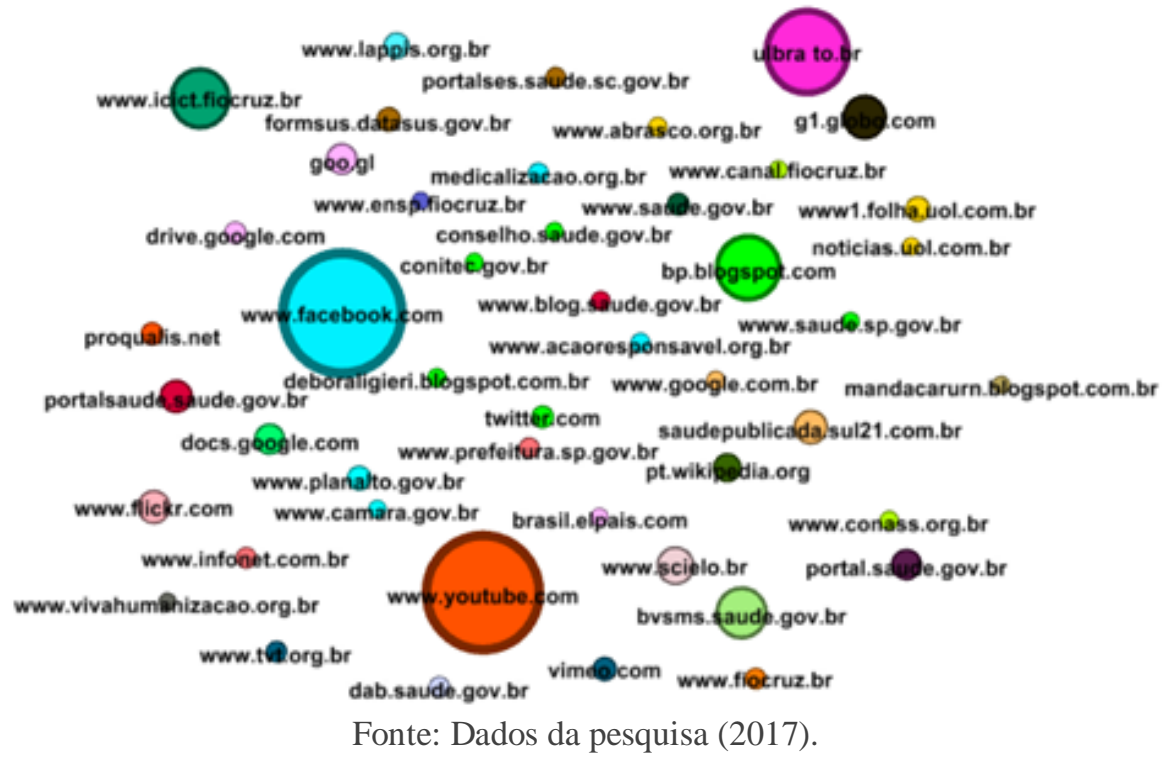


A partir da Figura 6 percebe-se melhor a distribuição dos distintos outlinks dentro da rede, na figura estão representados um total de 45 outlinks, sendo que aqueles que apresentam um tamanho maior, tem mais aparições nas postagens da RHS e suas cores remetem ao agrupamento a que fazem parte nos dados da rede, em sua maioria os nós apresentados tem um papel central em seu agrupamento. Fica aqui evidente, pelo tamanho do nó, a recorrência de citações a links do Youtube, Facebook e Ulbra, sendo esses os três maiores grupos que se destacam em meio aos outros nós.

Percebe-se que a visualização da rede corrobora com os dados apresentados anteriormente, gerando um modo visual de perceber a análise webométrica apresentada.

Já a rede a partir dos backlinks (Figura 7) se mostra muito mais densa, contendo um total de 25.910 nós e 45.170 arestas, além de 121 agrupamentos; dentre os agrupamentos existem dez deles que são mais expressivos e ocupam $98,58 \%$ da rede, os restantes $1,42 \%$ da rede se apresentam granulados e distantes do centro da rede.

Figura 7 - Rede de Backlinks

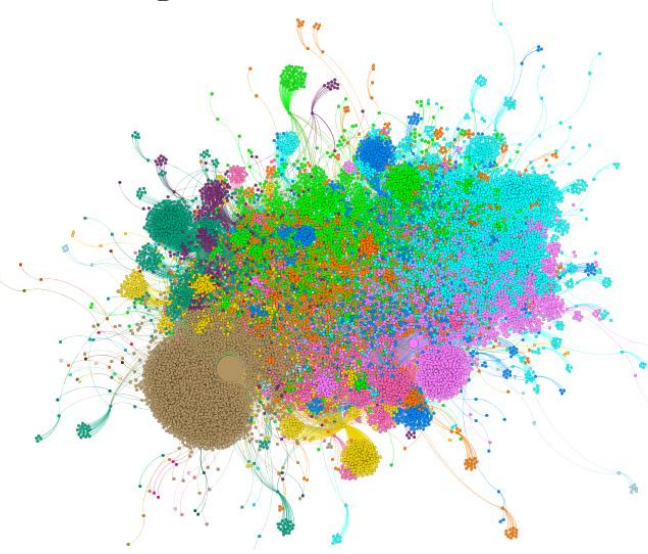

Fonte: Dados da pesquisa (2017).

Tal como apresentado anteriormente, o domínio Facebook.com (Quadro 4) é o que mais gera backlinks. Ao isolarmos o cluster referente a esse domínio que tem uma alta representatividade nos dados e na rede, temos a visão do quão importante ele se torna nesse contexto. Somente esse cluster representa $39.79 \%$ da rede contendo 10.310 nós e 10.644 arestas. 


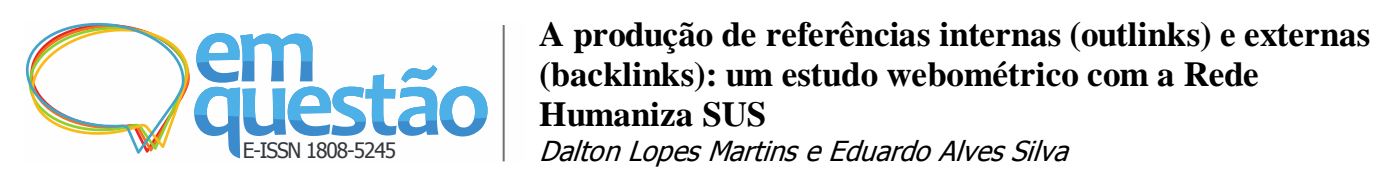

Figura 8 - Rede de Backlinks (Facebook.com cluster) da RHS

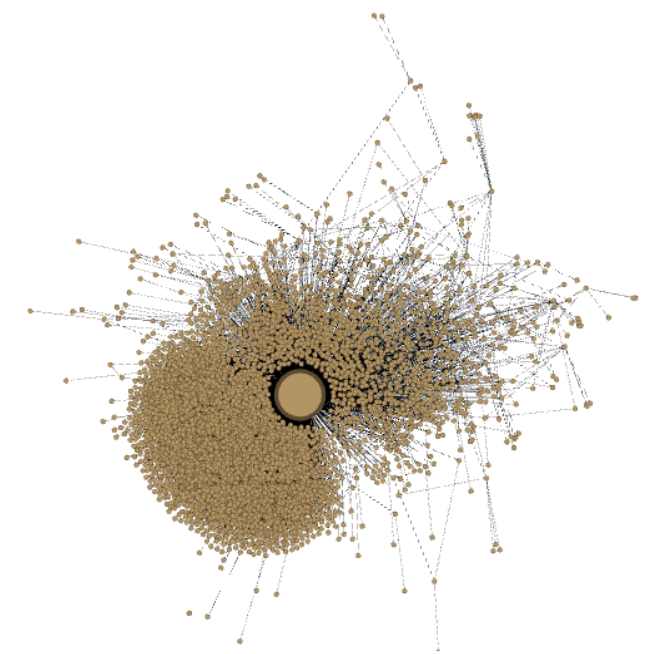

Fonte: Dados da pesquisa (2017).

\section{Conclusão}

A utilização de técnicas da webometria se mostrou um método de análise completo e de fácil compreensão no ambiente o qual a RHS se coloca, sendo um ativo da rede que tem por objetivo agregar toda uma comunidade e se fazer visível perante o vasto contexto web, trazendo informações e a colaboração entre um coletivo focado em um determinado tema.

Ao se fazer uso da análise de redes sociais como uma camada de análise relacionada a webometria, alcançou-se um resultado bastante compreensivo a respeito da importância do estudo de links, uma vez que a partir do mesmo é possível analisar, visualizar e tomar ações para que a RHS passe a ter mais impacto no ambiente em que se encontra.

As hipóteses foram parcialmente validadas, dado que se observou as mídias sociais tanto funcionando como recursos para trazerem usuários de volta para a rede e circularem informação, quanto como referenciais para a produção de conteúdos dos próprios usuários. Os sites de instituições de mérito na área foram bem utilizados pelas duas dinâmicas de links, mostrando como a rede é tanto percebida como importante fonte de informação por essas instituições, quanto essas instituições são acessadas e referenciadas pelos usuários da rede.

Foi possível compreender parte da dinâmica de apropriação e produção social da informação, tais como o papel do webmail como ferramenta de socialização da informação em redes privadas de contatos entre usuários e o 
papel do Facebook como fonte de informação de citação e referência para a produção da textualidade dos posts de blog criados na RHS. Compreender essas dinâmicas é compreender os modos de funcionamento e circulação da informação em rede, entendendo novos meios e ambientes por onde uma política pública se constitui rede quando disponível na internet a partir de ferramentas como a RHS. Possibilidades de uso dessas informações para novas estratégias de ativação e socialização em rede podem ser pensadas a partir dos resultados atuais.

Percebe-se na RHS a vivência de uma nova dinâmica de produção de informação relevante para área da saúde, descentralizada, colaborativa e em rede, sendo vista como referência significativa pela área. Vale reforçar que essa dinâmica de produção pode influenciar de forma significativa os próprios processos sociais de apropriação e produção da política pública de saúde em rede. Entender como esses impactos podem se dar e desenvolver novas formas de análise de políticas por meio da compreensão da dinâmica de funcionamento das redes sociais é um desafio para pesquisas futuras que se pretende apontar a partir deste trabalho.

\section{Referências}

ALCÂNTARA, Quézia.; TUZZO, Simone Antoniaci. O impacto das novas tecnologias midiáticas na cidadania e na educação. Comunicação \& Informação, v. 17, n. 1, p. 160-176, 2014.

https://doi.org/10.5216/cei.v1i1.31210

ALMIND, Tomas C.; INGWERSEN, Peter. Informetric analyses on the world wide web: methodological approaches to 'Webometrics'. Journal of Documentation, v. 53, n. 4, p. 404-426, 1997.

BJÖRNEBORN, Lennart. Small-world link structures across an academic web space: a library and information science approach. PHD dissertation. Copenhagen, DK: Departament of Informations Studies, Royal School of Library and Information Science, 2004.

BJÖRNEBORN, Lennart; INGWERSEN, Peter. Toward a basic framework for webometrics. Journal of the Association for Information Science and Technology, v. 55, n. 14, p. 1216-1227, 2004. https://doi.org/10.1002/asi.20077

GOUVEIA, Fabio Castro. Novos caminhos e alternativas para a webometria. Em Questão, v. 18, n. 3, p. 249-261, 2012. 
MARTINS, Dalton Lopes; SILVA, Eduardo Alves. Apropriação social de redes de relacionamento: a webometria como ferramenta de percepção da produção de referenciais internas (outlinks) e externas (backlinks). In: ENCONTRO BRASILEIRO DE BIBLIOMETRIA E CIENTOMETRIA, 6., 2018, Rio de Janeiro. Anais... Rio de Janeiro: UFRJ, 2018, p. 226-233. Disponível em: https://ebbc.inf.br/ebbc6/docs/6EBBC2018v2018_07_27.pdf. Acesso em: 20 dez. 2018

MARTELETO, Regina Maria. Análise de redes sociais: aplicação nos estudos de transferência da informação. Ciência da Informação, v. 30, n. 1, p.71-81, 2001.

MORIGI, Valdir Jose; MASSONI, Luis Fernando Herbert; SENA, Jocelaine Rodrigues. Memórias virtuais da cidade nas redes sociais: as ruas de Porto Alegre no Facebook. Pesquisa Brasileira em Ciência da Informação e Biblioteconomia, v. 11, n. 1, p. 33-43, 2016.

SANTOS, José Carlos Santos. Informação, democracia digital e participação política: uma breve revisão teórico-analítica. Em Questão, v. 19, n. 2, p. 195216, 2013.

SILVA, Roberto Bitencourt da. Mídias sociais e política: as jornadas de junho no Facebook do PT. Comunicação \& Informação, v. 16, n. 2, p. 53-71, 2013. https://doi.org/10.5216/c\&i.v16i2.27465

SILVA, Ilaydiany Oliveira. Webometria e a análise de redes sociais. Revista ACB: Biblioteconomia em Santa Catarina, v. 21, n. 2, p. 294-308, 2016.

VANTI, Nadia. Os links e os estudos webométricos. Ciência da Informação, v. 34, n. 1, p. 78-88, 2005. https://doi.org/10.18225/ci.inf..v34i1.1104

VANTI, Nadia. Links hipertextuais na comunicação científica: análise Webométrica dos sítios acadêmicos latino-americanos em Ciências Sociais. 2007. 299 f. Tese (Doutorado em Comunicação e Informação) - Curso de Programa de Pós-Graduação em Comunicação e Informação, Universidade Federal do Rio Grande do Sul, Porto Alegre, 2007.

WASSERMAN, Stanley; FAUST, Katherine. Social Network Analysis: Methods and applications. Cambridge, Reino Unido: Cambridge University Press, 1994.

WRIGHT, Alex. Forgotten forefather: Paul Otlet. 2003. Disponível em: $<$ http://boxesandarrows.com/forgotten-forefather-paul-otlet/>. Acesso em: 10 set. 2018. 


\title{
The production of internal (outlinks) and external (backlinks) references: a webometric study with Humaniza SUS Network
}

\begin{abstract}
Understanding social appropriation in a web environment brings benefits to the central point of this appropriation and for those who are impacted by this context, the use of webometrics has been increasingly used as a way to measure the impact that a website or a social network generates in the case of the humaniza SUS network (RHS), a study was made of outlinks that are presented as external addresses in the network posts, and the backlinks that generate accesses from external sites, together with the use of webometrics, was made use of the methodology of social network analysis (ARS) that implements a visual aspect to the data in order to facilitate its understanding, and demonstrating how the humaniza SUS network is perceived as a source of information by different institutions, many of them in the health area, just as these institutions are accessed and referenced by the users of the network.
\end{abstract}

Keywords: Humaniza SUS Network. Appropriation. Social. Webometrics. Social Network Analysis.

Recebido: 24/09/2018

Aceito: 03/12/2018

\footnotetext{
${ }^{1}$ Disponível em: 〈https://ebbc.inf.br/ebbc6/docs/6EBBC2018v2018_07_27.pdf\#page=226>. Acesso em: 20 nov. 2018.

${ }^{2}$ Disponível em: <www.redehumanizasus.net>. Acesso em: 12 dez. 2018.

${ }^{3}$ Disponível em: < http://portalms.saude.gov.br/>. Acesso em: 12 dez. 2018.

${ }^{4}$ Disponível em: < https://portal.fiocruz.br/>. Acesso em: 20 jan. 2019.

${ }^{5}$ Disponível em: < http://bvsms.saude.gov.br/>. Acesso em: 20 jan. 2019.
} 